\title{
ESTUDO DOS EVENTOS EXTREMOS DE PRECIPITAÇÃO OCORRIDOS EM 2009 NO ESTADO DO PARÁ
}

\author{
RENATA SILVA DE LOUREIRO ${ }^{1}$, JACI MARIA SARAIVA ${ }^{2}$, IVAN SARAIVA $^{2}$, RENATO CRUZ \\ SENNA $^{2}$, AMNE SAMPAIO FREDÓ $^{1}$ \\ ${ }^{1}$ Universidade do Estado do Amazonas (UEA), Instituto Nacional de Pesquisas da Amazônia (INPA), \\ Manaus, AM, Brasil \\ ${ }^{2}$ Sistema de Proteção da Amazônia (SIPAM), Manaus, AM, Brasil
}

renatasloureiro@yahoo.com.br, jaci.saraiva@sipam.gov.br, ivansaraiva@gmail.com,renato.senna@sipam. gov.br, bramnesf@gmail.com

Recebido Setembro de 2013 - Aceito 15 de outubro de 2014

\begin{abstract}
RESUMO
A importância da previsão meteorológica para períodos muito curtos de tempo (nowcasting) tem crescido nos últimos anos, devido ao aumento considerável de desastres naturaiscomo enchentes, deslizamentos de terra, rajadas de vento, etc. Nesse contexto, o uso do equipamento radar meteorológico tem grande utilidade operacional, na detecção e acompanhamento de fenômenos meteorológicos adversos de curto prazo. A Região Metropolitana de Belém (RMB) é uma das regiões mais populosas da Amazônia. Nos últimos anos vem apresentando uma ocupação urbana desordenada, o que talvez possa ser uma das causas dos problemas que a população sofre com os alagamentos e inundações. Os eventos extremos ocorridos durante o ano de 2009 foram identificados e analisados com o uso do TITAN (Thunderstorm Identification, Tracking, Analysis and Nowcasting). Os resultados mostram que $66 \%$ dos eventos extremos ocorreram na estação chuvosa, sendo devido a três principais sistemas meteorológicos, a Zona de Convergência Intertropical (ZCIT), a interação desta com a Zona de convergência do Atlântico Sul (ZCAS) e as Linhas de Instabilidade (LI) em sua maioria originadas na brisa marítima. Os resultados mostram também que os sistemas de menor escala, como as LI, apresentam severidade maior por provocar altas taxas de precipitação horária.

Palavras-chaves: Tempestades severas, Radar Meteorológico, Previsão de Tempo, Evento Extremo.
\end{abstract}

\section{ABSTRACT: STUDY OF SEVERE RAINFALL EVENTS OCCURRING IN 2009 IN THE STATE OF PARÁ}

The importance of weather forecasts for very short periods of time (nowcasting) has increased in recent years due to the considerable increase in natural disasters such as floods, landslides, wind gusts, etc. In this context, the use of weather radar equipment has great operational utility in the detection and monitoring of adverse short notice meteorological phenomena. The metropolitan region of Belém (RMB) is one of the most populous regions of the Amazon. In recent years unplanned urban occupation is taking place, being probably one factor that causes troubles for the population that suffers due to downpours and flooding. The 2009 year extreme events were identified and analyzed using the TITAN (Thunderstorm Identification, Tracking, Analysis and Nowcasting). The results show that $66 \%$ of severe events have occurred during the rainy season, which was due to three major weather systems: the Intertropical Convergence Zone (ITCZ), its interaction with the South Atlantic Convergence Zone (SACZ) and Squall Lines (LI) originated mostly from the sea breeze. The results also show that smaller-scale systems, like the lines, have higher severity because they cause higher rates of hourly precipitation.

Keywords: Severe Storms, Weather Radar, Weather Forecast, Extreme Event. 


\section{INTRODUÇÃO}

Na região norte, o estado do Pará é o mais populoso, com 7.581.051 habitantes. Sua capital é o município de Belém que reúne em sua região metropolitana cerca de 2.763.649 habitantes, composta pelos municípios de Ananindeua, Marituba, Benevides, Santa Isabel do Pará e Santa Bárbara do Pará. A Região Metropolitana de Belém sozinha concentra aproximadamente $40 \%$ da riqueza e $35 \%$ da população de todo o estado, segundo o Instituto Brasileiro de Geografia e Estatística (IBGE, 2010).

A região Amazônica apresenta regime de precipitação modulado por sistemas atmosféricos dinâmicos de micro, meso e grande-escalas. Nos últimos anos, pesquisas procuram entender a relação entre a convecção e a grande-escala. A convecção é um importante mecanismo de aquecimento da atmosfera tropical, do ponto de vista de Grimm e Silva Dias (1995) e Gandu e Silva Dias (1998) a fonte de calor tropical da Amazônia (convecção) e sua extensão para sudeste, conhecida como Zona de Convergência do Atlântico Sul (ZCAS) estão acopladas com outras fontes de calor tropical, como a do Pacífico e da África, de modo que, perturbações impostas por uma delas afeta a outra na escala de tempo de oscilações intrasazonais.

Os principais sistemas atmosféricos que atuam no tempo e no clima da região Amazônia são: a Zona de Convergência Intertropical (ZCIT), Zona de Convergência do Atlantico Sul (ZCAS), Linhas de Instabilidade (LI), Complexos Convectivos de Mesoescala (CCM), Ondas de Leste e as Brisas terrestre e marítima. Em Cohen et al. (2009) encontra-se uma coletânea de artigos sobre a atuação desses sistemas sobre a América do Sul, incluindo os resultados mais relevantes acerca dos sistemas atmosféricos responsáveis pela precipitação na região Amazônica. Esses sistemas, geralmente, estão associados a precipitações, ou seja, influenciam diretamente a estação chuvosa, descritos em trabalhos pioneiros como: Nobre (1983), Rao e Hada (1990), Rao et al. (1996), Marengo (2001), entre outros.

Molion $(1987,1993)$ conceitua as circulações de grande e meso-escala que atuam na Amazônia e os processos dinâmicos que organizam e geram a precipitação. Em seus estudos o autor divide a precipitação em três tipos: 1) convecção diurna resultante do aquecimento da superfície e condições de grandeescala favoráveis; 2) linhas de instabilidade originadas na costa N-NE do litoral do Atlântico, e finalmente, 3) aglomerados convectivos de meso e de grande escala, associados com a penetração de sistemas frontais na região S/SE do Brasil e interagindo com a região Amazônica.

Dentre os sistemas de mesoescala atuantes, destacam-se as Linhas de Instabilidade, amplamente estudada por Cohen (1995). Estas linhas, por apresentarem características diferentes (tempo de vida e velocidade de propagação, por exemplo), receberam denominações específicas vinculadas às diferenças observadas.

A região Amazônica é caracterizada por apresentar clima quente e úmido durante todo o ano e a precipitação é o parâmetro com maior heterogeneidade e variabilidade espaço-temporal. Por esta razão, essa região apresenta duas estações do ano que são divididas em dois períodos: o chuvoso (de fevereiro a maio) e o menos chuvoso (de setembro a novembro). Os outros meses do ano são considerados meses de transição.

O estudo do comportamento e estrutura de sistemas tropicais de mesoescala e de grande escala, realizados anteriormente, como em Houze e Rapparport (1984), Gamache e Houze (1982), Houze (1977), Betts (1978), Chong et al. (1987), Drosdowsky e Holland (1987), entre outros, basearam-se em dados coletados em experimentos de campo realizados em regiões tropicais. Por não existir um conjunto de dados suficiente para uma melhor compreensão desses sistemas tropicais, uma fonte de informação indispensável, seria a utilização dos dados de radar meteorológico, para preencher essa lacuna e auxiliar na compreensão da atmosfera tropical e suas interações, bem como prever com eficácia desastres naturais, que vem acontecendo com frequência em virtude de alterações climáticas (Tomasella et al.2010).

Nas últimas décadas, os eventos extremos têm causado constantes impactos em inúmeras cidades brasileiras. Estes podem ocorrer na forma de ondas de calor, períodos de estiagem, inundações, deslizamentos, etc. Certamente, as estiagens, as inundações e os deslizamentos são os desastres naturais mais frequentes no país. Estes últimos ganharam maior destaque na mídia eletrônica e impressa devido aos impactos socioambientais ocasionados nas grandes áreas urbanas, a citar: elevado número de mortes, feridos e desabrigados, proliferação de doenças, perdas econômicas onerosas, impactos ao meio ambiente, dentre outros. Tais impactos estão fortemente relacionados com a maior exposição da população a riscos diversos, acarretando em uma situação de vulnerabilidade cada vez mais recorrente. É bem verdade que o fenômeno da urbanização desordenada presente nas grandes cidades brasileiras contribui ainda mais para um aumento nos impactos ocasionados por episódios de precipitações extremas, as quais atingem, principalmente, as populações mais vulneráveis.

Nesse sentido, este trabalho objetiva identificar e descrever as características das chuvas intensas ocorridas na região de cobertura do Radar Meteorológico de Belém-PA, que causaram impactos socioambientais para a população. Para isso, as seguintes etapas serão desenvolvidas: 1-Identificação e seleção das chuvas intensas; 2- Estudo de caso utilizando dados de radar meteorológico descrevendo as características das chuvas intensas (análise de refletividade e estimativa de precipitação acumulada). 


\section{DADOS E MÉTODO}

\subsection{Dados}

O conjunto de dados utilizados nesta pesquisa engloba dados de superfície, dados do radar meteorológico localizado em Belém, dados diários de radiação de onda longa (ROL), dados de METAR, bem como imagens de satélite geoestacionário e dados do impacto das tempestades na cidade, através de informações jornalísticas.

\subsubsection{Dados de precipitação medidos em estações de superfície}

Os dados de precipitação foram obtidos juntos ao Instituto Nacional de Meteorologia (INMET), utilizaram-se dados de cinco estações no ano de 2009 que estão localizadas em Belém (Estação No 82191, Lat. $-1.43^{\circ}$, Lon. $-48.43^{\circ}$ ), Breves (Estação No 82188, Lat. -1.68 ${ }^{\circ}$, Lon. -50.48 ${ }^{\circ}$ ), Cametá (Estação $\mathrm{N}^{0} 82263$, Lat. $-2.25^{\circ}$, Lon. $-49.5^{\circ}$ ), Soure (Estação No 82141, Lat. $-0.73^{\circ}$, Lon. $-48.52^{\circ}$ ) e Tracuateua (Estação $N^{\circ} 82145$, Lat. $-1.07^{\circ}$, Lon. $-46.9^{\circ}$ ), conforme mostra a Figura 1. O critério para escolha das estações foi estar na área de cobertura do radar meteorológico instalado no município de Belém.

\subsubsection{Dados de radar meteorológico}

Neste trabalho também foram utilizados os dados volumétricos do radar banda $\mathrm{S}$ do Sistema de Proteção da
Amazônia (SIPAM). A varredura volumétrica serve para realizar medidas com boa precisão da área com raio de $240 \mathrm{~km}$, executando 17 elevações a partir da superfície e resolução temporal de 12 minutos. Esse modo permite a visualização em diversos níveis de altura para identificação da estrutura dos sistemas atmosféricos.

\subsubsection{Dados de ROL}

Os dados diários de radiação de onda longa (ROL) derivados dos satélites meteorológicos de órbita polar foram utilizados neste trabalho para caracterizar a atividade convectiva tropical. Os dados de ROL encontram-se dispostos numa grade regular com resolução de $2,5^{\circ}$ x $2,5^{\circ}$ de latitude x longitude.

\subsubsection{Dados de METAR}

Para identificação da intensidade da chuva e para verificar se as mesmas foram acompanhadas de rajadas de ventos e trovoadas utilizou-se informações meteorológicas horárias de superfície do METAR (METeorological Aerodrome Report) do aeródromo de Belém.

\subsubsection{Imagens de satélite}

Para identificação do ambiente atmosférico foram utilizadas imagens do satélite GOES 10 obtidas da página do Centro de Previsão e Estudos Climáticos - CPTEC (http:// satelite.cptec.inpe.br/acervo/goes.formulario.logic).

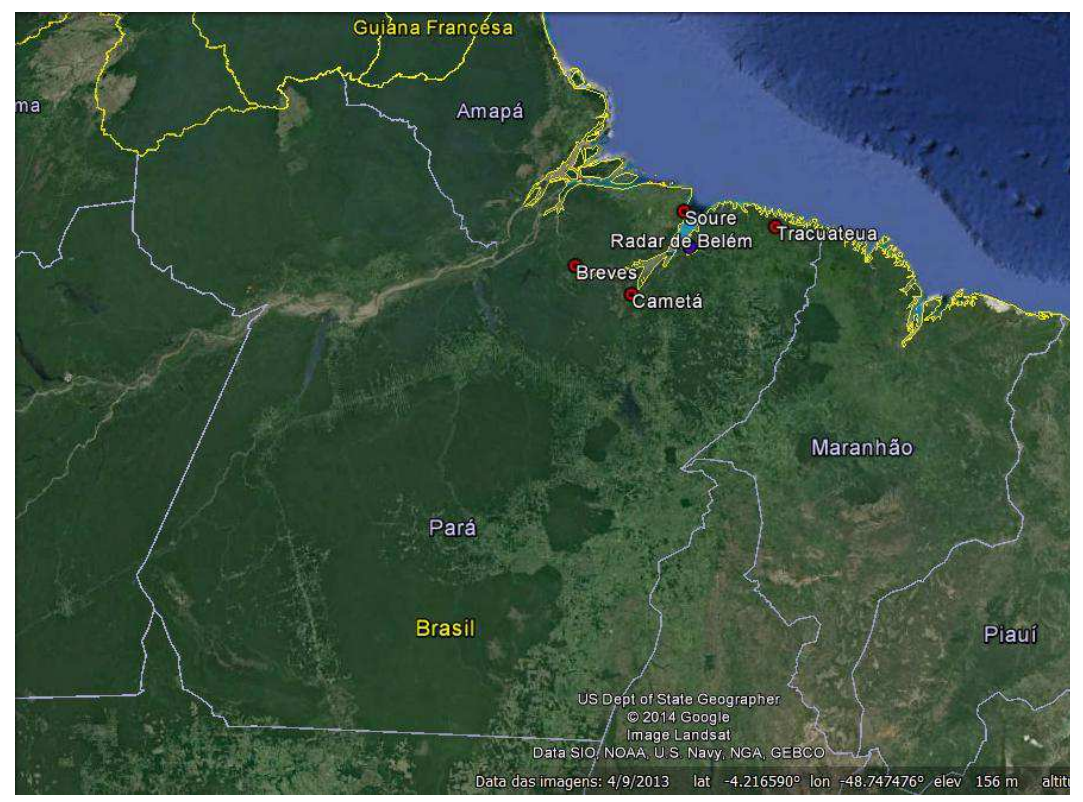

Figura 1 - Área selecionada para o estudo representativo ao raio de alcance de $240 \mathrm{~km}$ a partir do radar meteorológico de Belém. 


\subsubsection{Informes jornalísticos}

Informações documentadas pelo jornal Diário do Pará foram utilizadas para constatar os impactos da chuva.

\subsection{Método}

\subsubsection{Definição de eventos significativos utilizando dados de precipitação observados}

Inicialmente foi feito um levantamento completo dos dados de precipitação ocorridas durante o ano de 2009, utilizando como critério para definir evento significativo todo o registro maior ou igual a $25 \mathrm{~mm}$ em um período de 24 horas, em pelo menos uma das cinco estações que se encontram na área de abrangência do radar.

A partir do critério escolhido foi encontrado um total de 201 amostras com registros superiores a $25 \mathrm{~mm}$, distribuídas aleatoriamente nas cinco estações meteorológicas localizadas na área de abrangência do radar (Figura 2). A este conjunto de amostras foi aplicado o método estatístico dos Percentis, ou análise dos "quantis", muito utilizado por Xavier et al. (2000) e selecionados os limiares de $34 \%$ e $66 \%$ com o objetivo de categorizar as mesmas em três classes, listadas abaixo: Intenso (25-33mm), Muito Intenso (33,1 - 48mm) e Extremamente Intenso ( $\geq 48 \mathrm{~mm})$. Para configurar um evento, o critério adotado foi escolher os casos em que a precipitação ocorreu em três ou mais estações. Assim, das 201 amostras (a partir de agora denominado amostras) resultaram em apenas 121 eventos. A distribuição sazonal e a relação entre número de amostras e de eventos é ilustrada na Figura 3. Para o caso de amostras/eventos superiores a $48,1 \mathrm{~mm}$ em 24horas, dos eventos classificados como extremamente intenso seis foram selecionados (quantil
$>66 \%$ ). Essa seleção de eventos esta em destaque na Tabela 1.

\subsubsection{Análise das tempestades utilizando dados de Radar}

As tempestades identificadas na Tabela 1 foram analisadas utilizando os dados de radar com a varredura de 240 $\mathrm{km}$ e o programa utilizado para gerar os campos analisados foi o TITAN (Thunderstorm, Identification, Tracking, Analysis and Nowcasting). Os dados de refletividade apresentam formato original em mvol, sendo necessário converte-los para MDV (Meteorological Data Volume). O formato MDV é um formato capaz de armazenar dados em grades de até três dimensões. Ele é altamente estruturado e possui habilidade para gerenciar múltiplos campos de dados em um único arquivo. O MDV requer o espaçamento constante de dados nos planos x-y para cada campo. O método usa como base as informações do radar em coordenadas cartesianas.

De acordo com Dixon e Wiener (1993) a aplicação Titan identifica células de tempestades em dados volumétricos de radar em coordenadas cartesianas e verifica a trajetória das células identificadas, fornecendo uma previsão de seu movimento. Essa aplicação identifica uma tempestade como sendo uma região contígua tridimensional de tal forma que certos parâmetros, como a refletividade, o volume, a altura, entre outros, satisfaçam determinadas condições. A configuração do TITAN nesta pesquisa utilizou o valor de $20 \mathrm{dBZ}$ como limiar de refletividade mínima. Para estudos utilizando radar meteorológico, os referidos autores propuseram três índices para definir a severidade das tempestades: fraco (30 - 44dBZ), moderado $(45-50 \mathrm{dBZ})$ ou severo $(>55 \mathrm{dBZ})$ que serão utilizados como parâmetros para discussão da distribuição dos valores de MAXCAPPI.

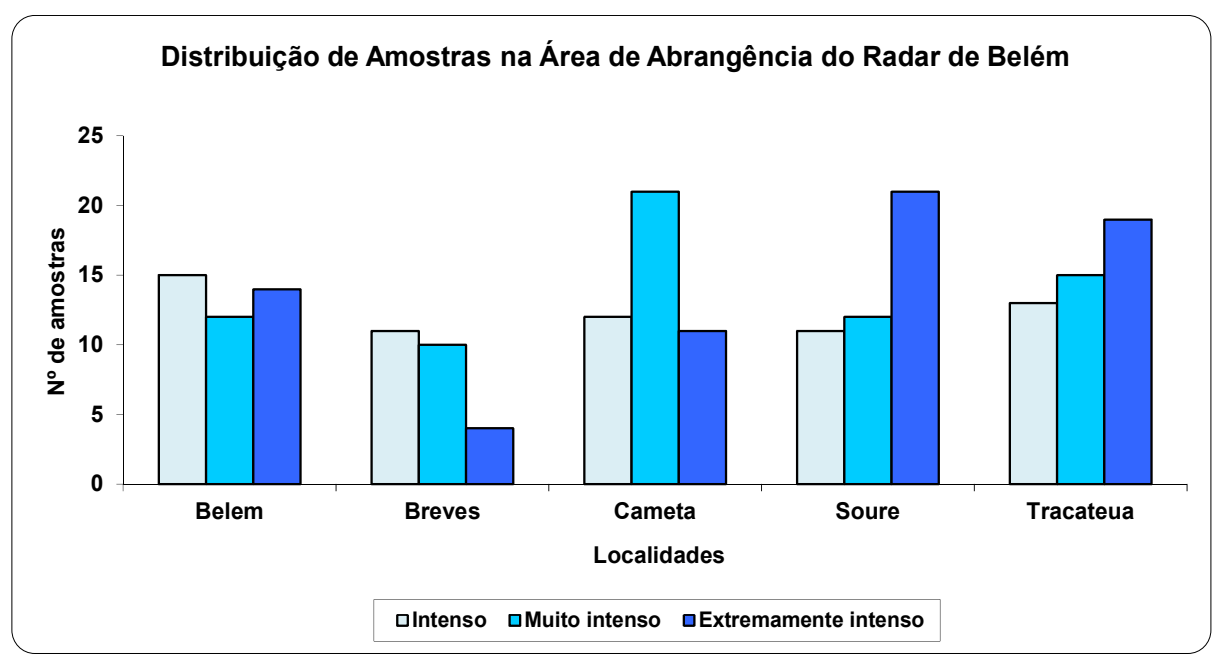

Figura 2 - Distribuição do $\mathrm{N}^{0}$ de amostras categorizadas para cada uma das estações dentro da área de abrangência do radar. 
Distribuição de amostras e eventos ao longo do ano

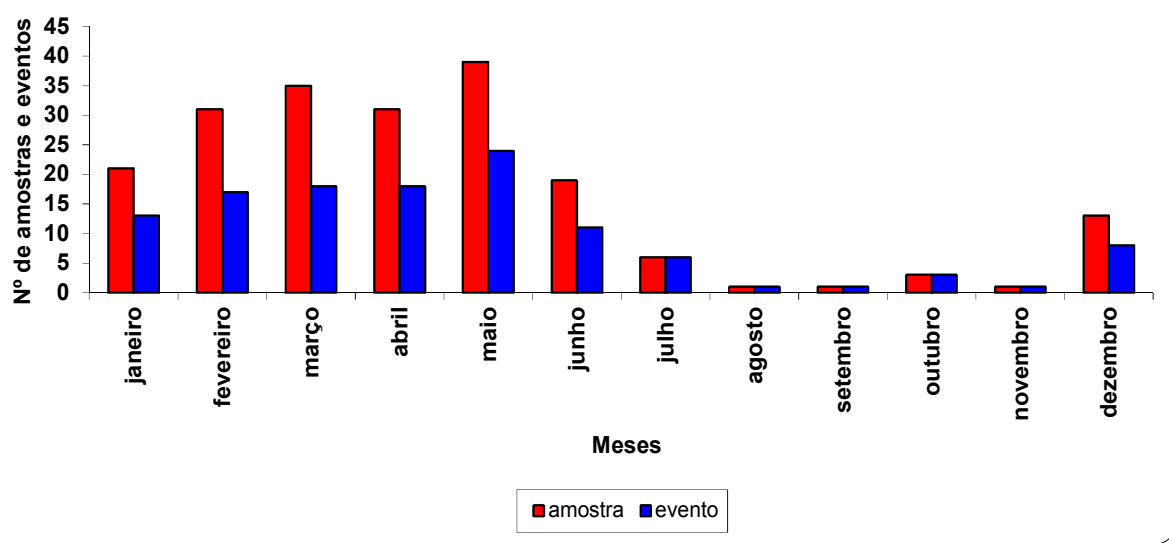

Figura 3 - Distribuição mensal do número de amostras e eventos no ano de 2009 ..

Nesta pesquisa só será apresentada a espacialização dos campos de MAXCAPPI e a precipitação acumulada do período de um dia. A precipitação estimada foi realizada a partir do produto CAPPI de $2 \mathrm{~km}$ e a relação $\mathrm{Z}-\mathrm{R}\left(\mathrm{Z}=315 \mathrm{R}^{1,20}\right)$ aplicada foi extraída de Tokay e Short (1996), que verificaram a distribuição das partículas de gotas de chuva e gelo na região tropical. Onde $\mathrm{Z}$ é a refletividade em $\mathrm{dBZ}$ medida pelo radar meteorológico, $\mathrm{R}$ a precipitação $\mathrm{em} \mathrm{mm} / \mathrm{h}$, a e b são as constantes da relação ZR.

\section{RESULTADOS E DISCUSSÃO}

\subsection{Ambiente atmosférico}

As análises dos padrões médios de Radiação de Onda Longa (ROL) indicam atividade convectiva intensa sobre a região equatorial, associados diretamente à atuação da Zona de Convergência Intertropical (ZCIT). Os valores abaixo de 240W/ $\mathrm{m}^{2}$ já são indicadores de convecção profunda (Kousky, 1988). Deste modo, grande parte da Amazônia Oriental apresenta valores entre $210 \mathrm{~W} / \mathrm{m}^{2}$ e $220 \mathrm{~W} / \mathrm{m}^{2}$. Quando observa-se os valores de ROL para o mês de maio de 2009 (Figura 4b), a Amazônia Oriental apresenta menores magnitudes em relação à climatologia (Figura 4a), ou seja, a convecção foi realçada sobre o Pará, pois os valores de ROL concentram-se em torno $\operatorname{dos} 200 \mathrm{~W} / \mathrm{m}^{2}$. Tal condição indica maior quantidade de nuvens convectivas, justificando os acumulados mensais de precipitação muito acima da normalidade, principalmente no litoral do Pará e Ilha de Marajó, conforme mostra a Figura 4.

As informações das análises de mesoescala apresentadas na Tabela 1 foram extraídas do Boletim de Monitoramento e Análise Climática - Climanálise. Nessa tabela observa-se a predominância das LI com horário de formação no período da tarde a partir da brisa marítima. As linhas têm a capacidade de modificar o ambiente por onde passam, resultado semelhante foi encontrado por Alcântara et al. (2011) utilizando um conjunto de nove anos de dados. Além de identificar o horário predominante à tarde, essa autora também identificou uma ocorrência média de LI, na costa norte do Brasil, a cada dois dias desse sistema na região, distribuídas com 54\% de LIC, 26\% de LIP1 e 20\% de LIP2, seguindo a classificação proposta por Cohen (1995). Estudos pioneiros de Hann's (1901), apud Asnani (1993) propõem que as precipitações na Amazônia apresentam três modelos conceituais que são: o modelo continental, com chuvas ocorrendo preferencialmente à tarde e início da noite; e o modelo marítimo, com ocorrência predominantemente à noite e de madrugada e por fim o modelo misto, quando apresenta situações diferentes dos dois anteriores. Uma discussão pertinente sobre o assunto enfatiza a existência de uma grande variedade de padrões que não se encaixam nos modelos definidos por Hann's, alguns autores chegaram a resultados contrários (Wallace, 1975 e Gray e Jacobson 1977) concluindo que muitas áreas continentais tropicais não mostraram uma chuva máxima durante o período da tarde associada com o máximo aquecimento da superfície, pelo contrário, observaram uma grande ocorrência de máximos de chuvas durante o período noturno.

Abordando a precipitação quanto ao seu tipo, de uma forma geral, pode-se classificar o tipo de chuva através de dois diferentes processos: chuva do tipo estratiforme e chuva do tipo convectiva. Essa classificação baseia-se principalmente em dois fatores: o valor da taxa de precipitação máxima e o tempo de duração observado em cada um destes processos. No caso da precipitação do tipo estratiforme (convectiva), a mesma apresenta grandes (pequenas) áreas de precipitação, permanece por longos (curtos) períodos de tempo e proporciona intensidade de precipitação média ou baixa (forte). 
Tabela 1 - Eventos com precipitação acumulada de 24 horas correspondentes a categoria Extremamente Intenso ( $\geq 48,1$ mm) nas estações meteorológicas localizadas na área de cobertura do Radar de Belém.

\begin{tabular}{|c|c|c|c|c|c|c|c|}
\hline \multicolumn{8}{|c|}{ PRECIPITAÇÃO ACUMULADA DE 24HS } \\
\hline DATA DO EVENTO & BELÉM & BREVES & CAMETÁ & SOURE & TRACUATEUA & SISTEMA ATMOSFÉRICO & HORÁRIO \\
\hline $19 / 01 / 09$ & 72,2 & - & - & - & - & LI & Tarde \\
\hline $24 / 01 / 09$ & - & - & - & 61,5 & - & LI & Noite \\
\hline $30 / 01 / 09$ & - & - & - & 67,3 & - & LI & Noite \\
\hline $01 / 02 / 09$ & & & & & 49,2 & Aglomerados Convectivos & Tarde \\
\hline 15/02/09 & - & 91,4 & 86,2 & 52,8 & - & ZCIT & Dia inteiro \\
\hline $16 / 02 / 09$ & - & - & - & - & 49,8 & ZCIT & Dia inteiro \\
\hline $18 / 02 / 09$ & - & - & - & - & 49,2 & Aglomerados Convectivos & Tarde \\
\hline 20/02/09 & - & - & - & 130,6 & 69,7 & LI & Tarde \\
\hline 21/02/09 & - & - & - & 75,5 & 118,2 & ZCIT & Dia inteiro \\
\hline $25 / 02 / 09$ & - & - & - & & 58,0 & LI & Tarde \\
\hline $02 / 03 / 09$ & - & - & - & 59,1 & - & LI & Tarde \\
\hline $04 / 03 / 09$ & & & & & 113,0 & LI & Tarde \\
\hline $15 / 03 / 09$ & 77,6 & 59,6 & 77,1 & 256,6 & 87,7 & $\mathrm{LI}+\mathrm{ZCIT}$ & Dia inteiro \\
\hline $16 / 03 / 09$ & - & - & - & 116,6 & - & $\mathrm{LI}+\mathrm{ZCIT}$ & Dia inteiro \\
\hline $22 / 03 / 09$ & - & - & - & 50,4 & - & $\mathrm{LI}$ & Tarde \\
\hline $23 / 03 / 09$ & - & - & - & 61,7 & 53,6 & ZCIT & Madrugada \\
\hline 26/03/09 & - & - & - & 61,4 & - & Aglomerados Convectivos & Noite \\
\hline $27 / 03 / 09$ & - & - & - & 66,9 & - & ZCIT & Manhã \\
\hline $30 / 03 / 09$ & 50,5 & - & 119,4 & & - & ZCIT & Tarde \\
\hline $31 / 03 / 09$ & 53,6 & 54,6 & - & 96,1 & - & ZCIT & Dia inteiro \\
\hline $01 / 04 / 09$ & - & - & - & 110,3 & - & $\mathrm{LI}$ & Tarde \\
\hline $04 / 04 / 09$ & - & - & - & 61,4 & - & LI & Manhã \\
\hline $09 / 04 / 09$ & - & - & - & 159,7 & 62,0 & LI & Tarde \\
\hline $11 / 04 / 09$ & - & - & 61,8 & - & - & Brisa & Tarde \\
\hline 15/04/09 & - & - & & 84,8 & - & LI & Tarde \\
\hline $16 / 04 / 09$ & - & - & 52,4 & - & - & LI & Tarde \\
\hline $25 / 04 / 09$ & 78,6 & - & - & - & - & Brisa & Tarde \\
\hline $27 / 04 / 09$ & 66,8 & - & - & 166,8 & 51,9 & ZCIT & Tarde \\
\hline $28 / 04 / 09$ & - & - & - & 53,8 & - & Aglomerados Convectivos & Noite \\
\hline 29/04/09 & - & - & 51,9 & - & - & LI & Tarde \\
\hline $01 / 05 / 09$ & - & - & - & - & 82,2 & $\mathrm{LI}$ & Madrugada \\
\hline $02 / 05 / 09$ & - & - & - & - & 51,8 & LI & Manhã \\
\hline 08/05/09 & - & - & - & - & 78,4 & Aglomerados Convectivos & Tarde \\
\hline $09 / 05 / 09$ & - & - & - & 77,4 & - & Brisa & Tarde \\
\hline 12/05/09 & - & - & - & - & 75,0 & Brisa & Tarde \\
\hline 13/05/09 & - & - & - & 53,2 & 55,4 & ZCIT & Tarde \\
\hline 14/05/09 & - & - & 87,1 & - & - & Aglomerados Convectivos & Manhã \\
\hline $23 / 05 / 09$ & 48,6 & 49,0 & - & - & 55,6 & $\mathrm{LI}+\mathrm{ZCIT}$ & Tarde \\
\hline $27 / 05 / 09$ & - & - & - & - & 80,8 & Brisa & Tarde \\
\hline $29 / 05 / 09$ & 63,1 & - & 73,4 & - & - & ZCIT & Dia inteiro \\
\hline $31 / 05 / 09$ & 64,8 & - & & - & - & $\mathrm{LI}$ & Noite \\
\hline $02 / 06 / 09$ & 54,0 & - & 103,2 & 140,6 & - & Aglomerados Convectivos & Tarde \\
\hline $06 / 07 / 09$ & 48,4 & - & - & - & - & Aglomerados Convectivos & Noite \\
\hline 18/10/09 & 61,9 & - & - & - & - & Brisa & Tarde \\
\hline 03/12/09 & 57,6 & - & - & - & - & Brisa & Tarde \\
\hline 11/12/09 & - & - & 100,8 & - & - & LI & Tarde \\
\hline $21 / 12 / 09$ & - & - & 54,7 & - & - & $\mathrm{LI}$ & Tarde \\
\hline
\end{tabular}




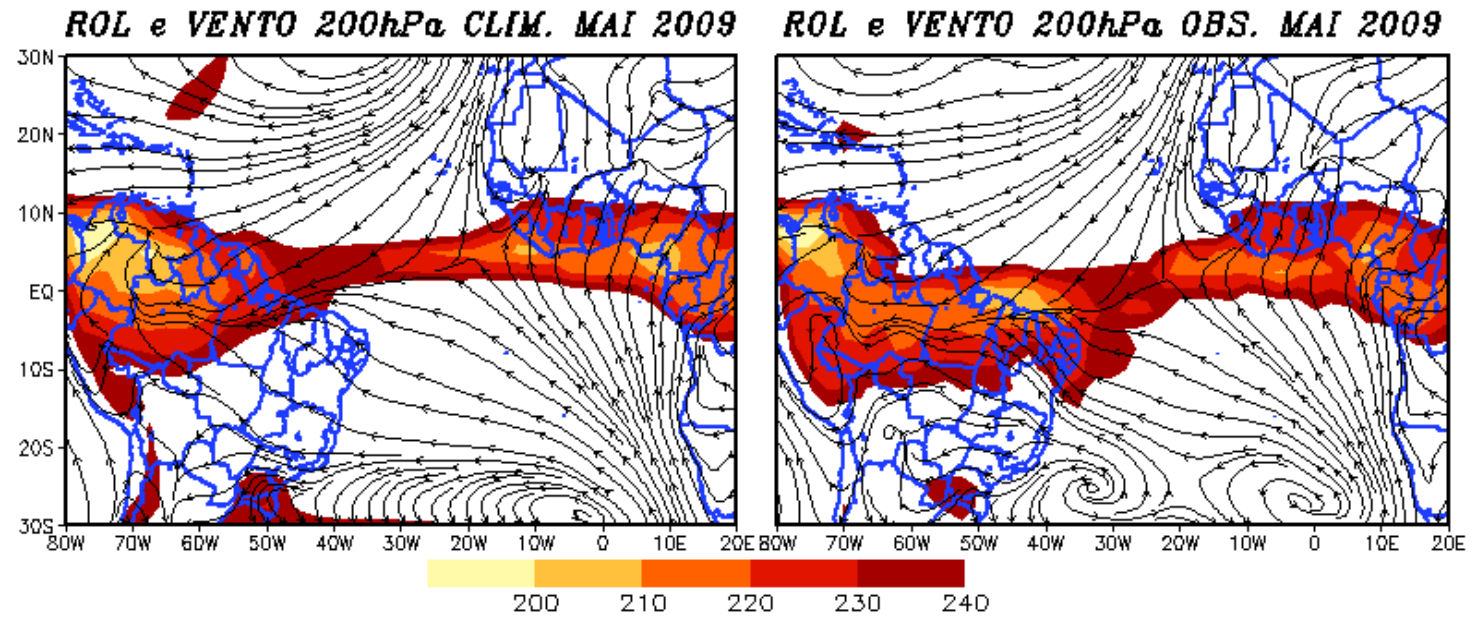

Figura 4 - (a) a climatologia de Radiação de Onda Longa (ROL) e vento horizontal em 200 hPa para o mês de maio e (b) observado para maio de 2009.

Os dados revelaram que o ano de 2009 foi atípico em relação à média dos anos anteriores, segundo os registros de precipitação do Instituto Nacional de Meteorologia (INMET). Nos municípios de Belém e Tracuateua, por exemplo, foram 3.463,6 e 3.284,3 milímetros de água, sendo a média climatológica, de 2.921,7 e 2.597,2 milímetros representando aproximadamente um acréscimo de 542 e 687 milímetros, respectivamente.

Os principais sistemas atmosféricos, responsáveis por esses elevados totais de chuva sobre a região, foram a ZCIT e as Linhas de Instabilidade (LI's), estas por sua vez foram documentadas em Climanálise (2009) em praticamente toda a estação chuvosa. Ainda segundo esse Boletim, durante o mês de janeiro foram caracterizados dezesseis episódios atuando ao longo da costa brasileira, quando a ZCIT atuou ao sul de sua posição climatológica. Sobre a região nordeste do Pará, em particular, a formação de LI's contribuiu para a ocorrência de chuvas acima da média histórica. Em fevereiro foram seis LI's, em março foram quatorze episódios, destaca-se o dia 15, no qual a atividade convectiva entre o nordeste do Pará e a costa norte do Nordeste foi mais intensa. Neste dia, em particular, a formação da LI foi intensificada pela convergência associada à ZCIT, favorecendo a ocorrência da chuva em Soure, na Ilha de Marajó (Tabela 1). Em abril, foram treze LI's, nove episódios no decorrer de maio e finalmente treze episódios em junho. Alguns registros dos impactos causados à população pelas fortes tempestades geradas por estes sistemas podem ser visualizados na Figura 5.

\subsection{Estudo de caso}

No estudo de eventos de chuvas intensas, enchentes, tempestades acompanhadas de granizos etc., normalmente a abordagem é feita seguindo duas vertentes: estudo de casos ou composição de um conjunto de casos. Sendo a primeira, escolhida para uma análise que fornece detalhamento sobre a evolução do ambiente atmosférico durante a ocorrência do evento extremo. Enquanto a segunda utiliza a informação coletiva de um conjunto de eventos associados a um mesmo fenômeno atmosférico para conhecer o ambiente que contribuiu para a sua ocorrência.

Nesta seção será mostrado o evento ocorrido em 23/05/2009, para tal será utilizado dados do radar meteorológico de Belém em conjunto com as imagens de satélite.

Durante o mês de maio as áreas de convecção associadas à ZCIT apresentaram grande variabilidade espacial, oscilando entre $5^{\circ} \mathrm{S}$ e o Equador, próximo à costa norte do Brasil. As imagens de satélite (Figura 6) indicam a presença da ZCIT bem estabelecida na faixa litorânea da região nordeste do Pará. No dia 23 a ZCIT (Figura 6a) esta visível sobre o Oceano Atlântico e favorece a intensificação da banda de nebulosidade com sentido meridional, que se desloca de leste para oeste em direção a capital Belém. As 1530UTC o radar já apresentava alguns pontos com refletividade de $35 \mathrm{dBZ}$ (Figura 7a).

Na sequência da evolução do sistema atmosférico tem-se no horário das 1700UTC (Figura 6b) a formação de nuvens com grande desenvolvimento vertical, e com atividade convectiva intensa. No radar a nebulosidade se organiza em forma de arco, a leste de Belém, com núcleos convectivos intensos, localizados a frente da parte estratiforme, com valores acima de 50dBZ, mostrando um grau de severidade moderado (Figura 7b). As 1800UTC (Figura 6c) é visível, o alinhamento meridional, a leste de Belém, de nuvens convectivas posicionadas na porção frontal e com uma banda de nuvens estratiformes na retaguarda deste alinhamento, atingindo sua maior intensidade meia hora depois as 1830UTC, com registro de chuva forte e trovoadas (Figura 6d-6e). Esta tempestade causou muitos danos, como interrupção do 


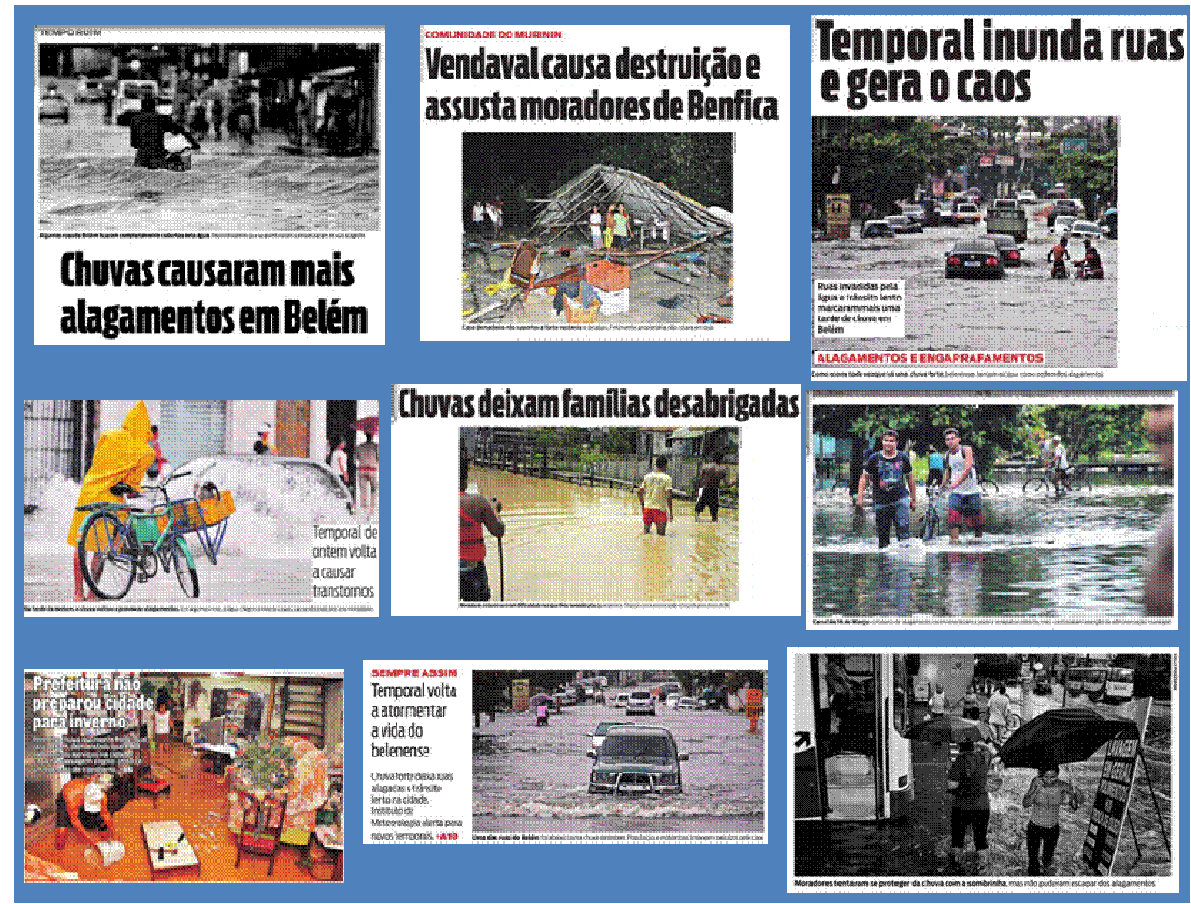

Figura 5 - Acervo jornalístico com os danos causados à população pelas chuvas intensas que ocorreram em 2009. Fonte: Diário do Pará (Maio/2009).

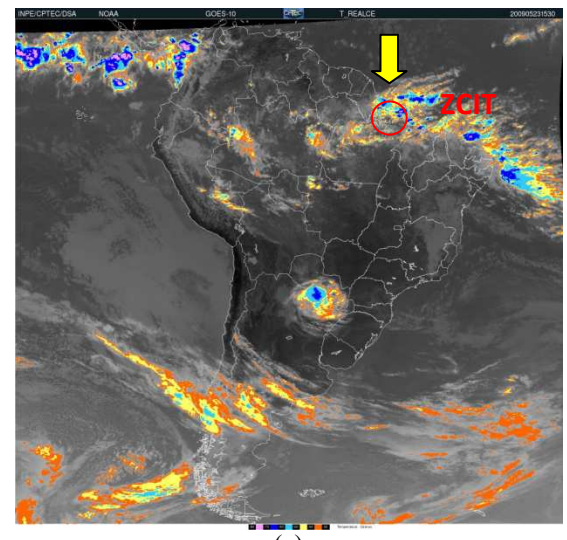

(a)

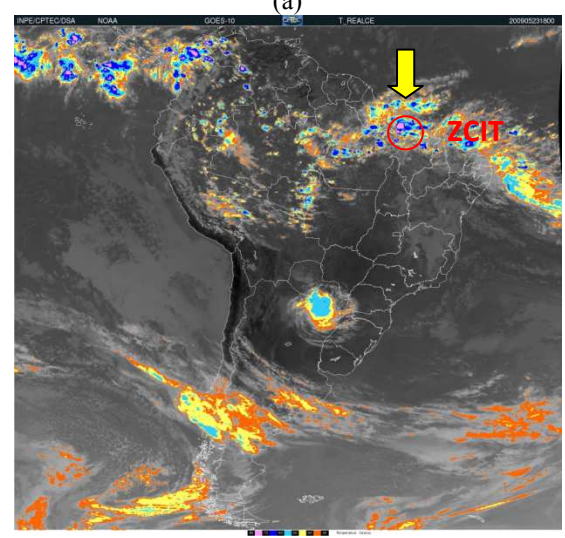

(c)

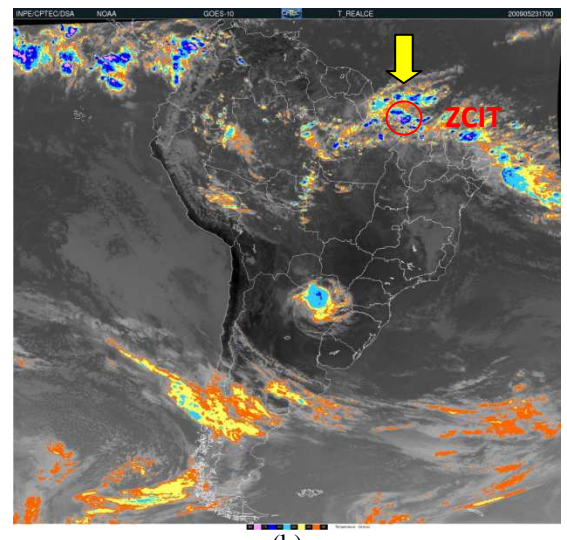

(b)

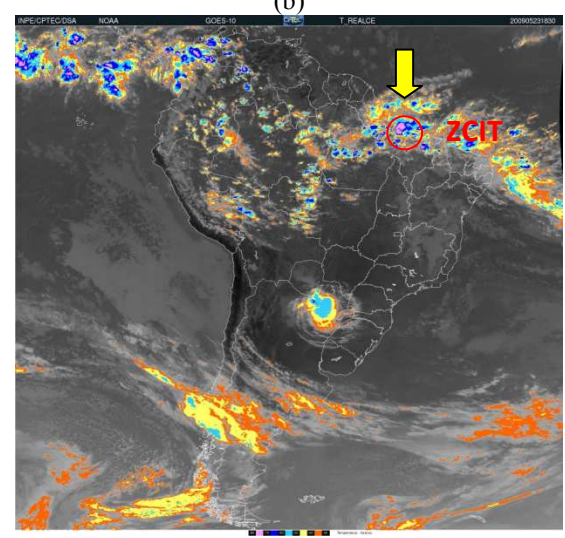

(d)

Figura 6 - Imagens do satélite geoestacionário GOES-10 mostrando: Caso ocorrido no dia 23 de maio. (a) 15:30 UTC, (b) 17:00 UTC, (c) 18:00 UTC e (d) 18:30 UTC.

Fonte: CPTEC/INPE. 


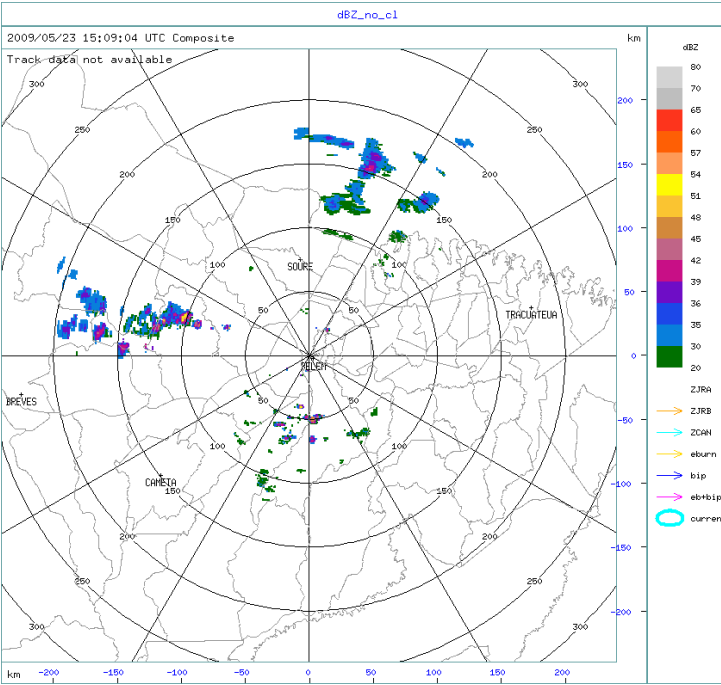

(a)

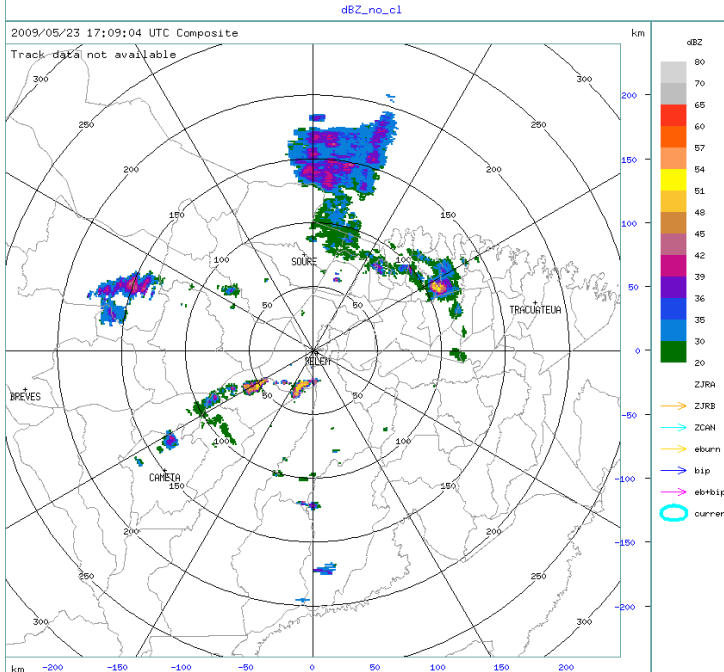

(c)

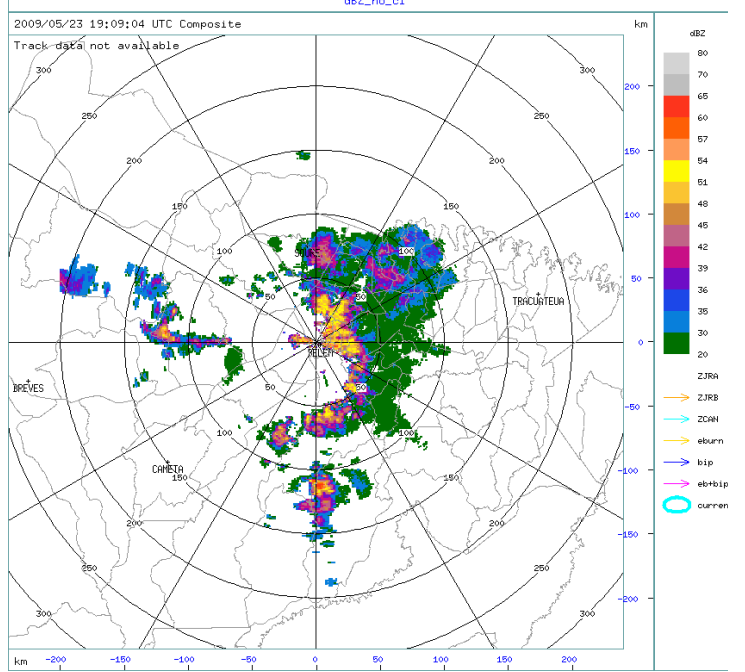

(e)

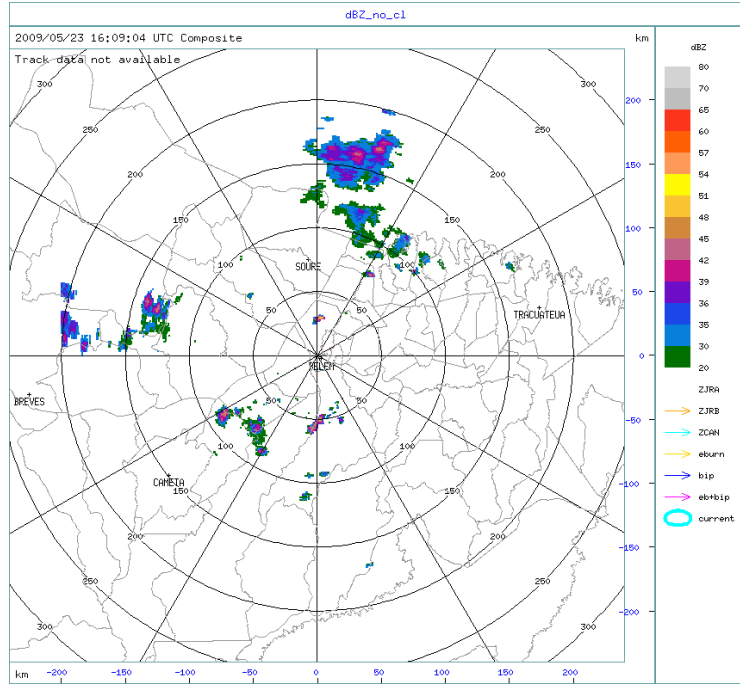

(b)

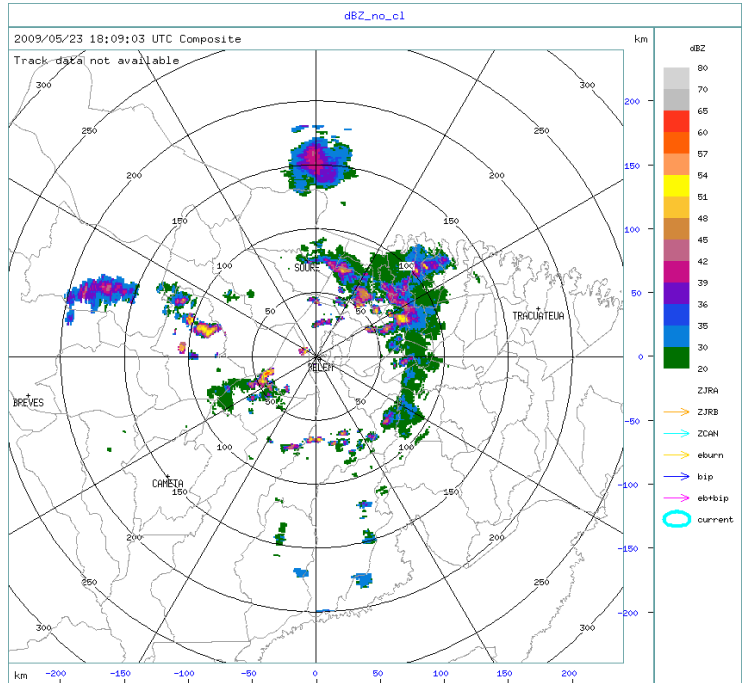

(d)

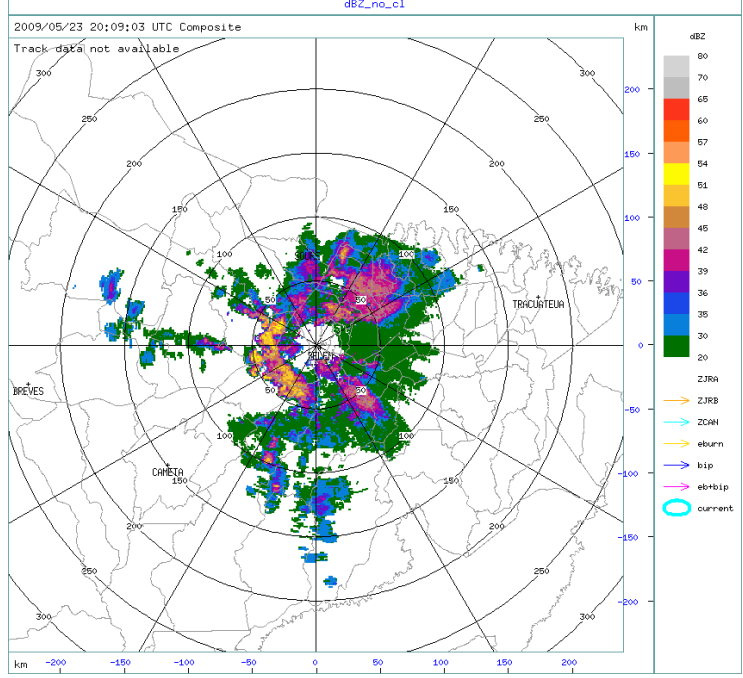

(f)

Figura 7 - Sequência horária do MAXCAPPI em 23/05/2009. Continua. 


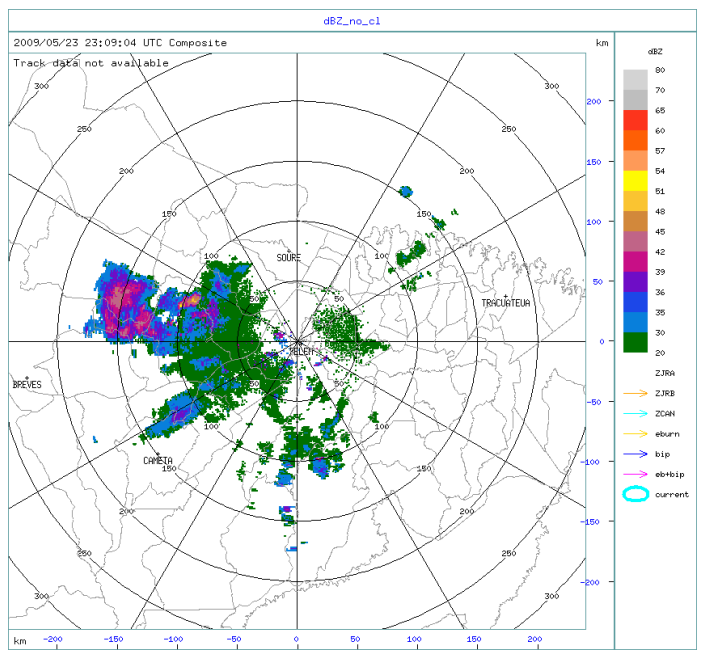

(i)

Figura 7 - Conclusão

fornecimento de energia elétrica, diversos pontos de alagamento, transbordamento de canais, queda de árvores, etc. A estação meteorológica de superfície de Belém registrou precipitação de 48,6mm em apenas uma hora, sendo acompanhada de trovoadas e rajadas de $20 \mathrm{KT}$, característico de nuvens de grande desenvolvimento vertical. Às 2009UTC (Figura 7f) na porção frontal do sistema, as células de tempestade permanecem muito intensas, mas com posicionamento a oeste do centro do radar e a parte estratiforme localizado na retaguarda do sistema ocupando uma área maior, ocasionando chuva leve e contínua. Nos horários seguintes verifica-se a dissipação e descaracterização do sistema, com redução dos valores de dBZ e dissipação das células convectivas que estavam localizadas a frente do alinhamento de nuvens. Os acumulados de chuva correspondentes a tal evento foram estimados pelo radar e são mostrados na Figura 8. Essa estimativa mostrou-se bem representativa dos valores registrados pelas estações meteorológicas de superfície (Tabela 1), onde os acumulados variaram de 40 a $60 \mathrm{~mm}$ em 24 horas, em todas as localidades que registraram chuva.

Resumidamente a tempestade apresentou intensidade variando de moderada ( $45-50 \mathrm{dBZ}$ ) a forte ( $>55 \mathrm{dBZ}$ ), as células com maior atividade convectiva ficaram localizadas sobre o município de Soure e Belém. A presença da ZCIT sobre a região e a formação da brisa marítima no período da tarde propiciou a formação desta linha que se deslocou na direção da capital do estado do Pará causando chuva intensa, rajadas de ventos e descargas atmosféricas.

\section{CONCLUSÕES}

Os eventos extremos registrados nas estações de superfície do estado do Pará no ano de 2009 ocorreram em sua maioria na estação chuvosa. Os acumulados mensais, nas cinco estações analisadas, apresentaram valores acima da média climatológica para o ano de 2009, com valores acima da média climatológica de $540 \mathrm{~mm}, 410 \mathrm{~mm}$ e $680 \mathrm{~mm}$ em Belém, Cametá e Tracuateua, respectivamente. Vale ressaltar que o valor diário mais significativo ocorreu em Soure (Ilha do Marajó) com um acumulado de $256,6 \mathrm{~mm}$, causado pela ZCIT. Porém, na contabilização dos casos de maior severidade das tempestades, observou-se em termos de quantidade, a maior ocorrência de sistemas de mesoescala, como as linhas de instabilidade. As espacializações dos campos de refletividade

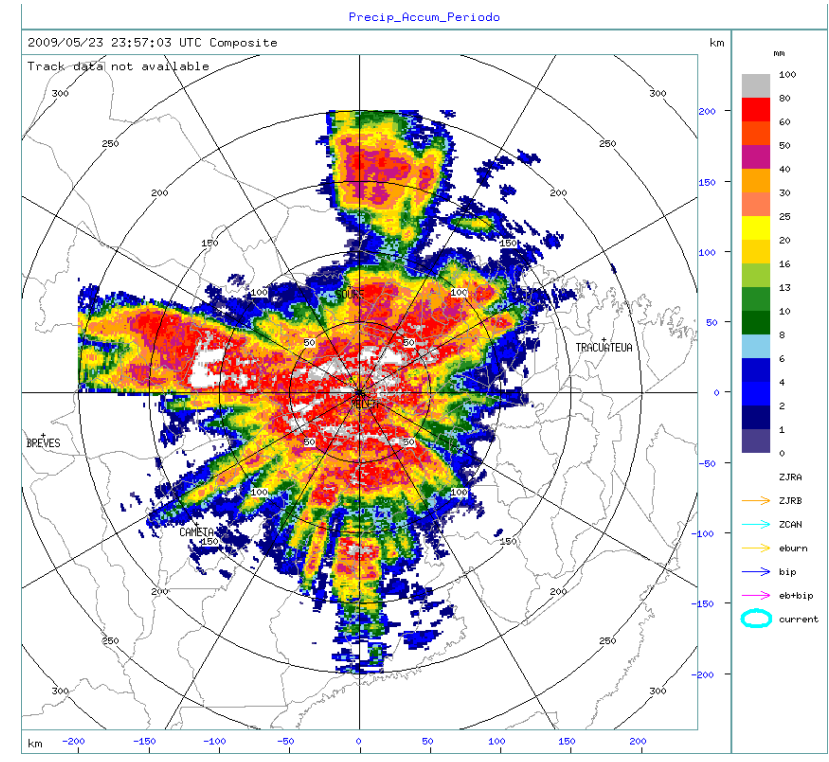

Figura 8 - Precipitação estimada pelo radar acumulada de 00 UTC até as 23:57 UTC do dia 23/05/2009. 
mostraram a formação das células de tempestades no litoral, corroborando com estudos anteriores. Na classificação utilizada neste estudo foram encontrados 69 eventos categorizados como Extremamente Intenso, ou seja, com precipitações maiores do que $48 \mathrm{~mm}$, os quais na maioria ocorreram por formação e/ou interação entre os sistemas meteorológicos de grande escala e a mesoescala. O uso do radar meteorológico mostrou-se uma potente ferramenta na identificação de formação de sistemas de menor escala dentro de um ambiente de grande escala favorável. O uso desta ferramenta na Amazônia oriental em especial próximo a costa, possibilitará em estudos futuros identificar o gatilho para estas formações, bem como, as variáveis que possibilitarão prognósticos de sua direção e velocidade de deslocamento.

Devido aos elevados totais pluviométricos registrados na estação chuvosa, ocorreram focos de alagamentos que foram responsáveis por problemas de circulação e comunicação urbana, entre eles: o transbordamento de canais que inundou as partes mais baixas da cidade, causando muitos prejuízos para os comerciantes locais. A identificação da severidade de tempestades, através do uso de dados do radar permitirão uma melhor compreensão dos processos envolvidos, desde a geração, intensificação e deslocamento dos sistemas. Também será possível alertar aos órgãos competentes com maior precisão, sobre a hora de ocorrência destas tempestades e sua severidade, assim como estimar a chuva associada à mesma.

\section{AGRADECIMENTOS}

Os autores agradecem a Financiadora de Estudos e Projetos - FINEP pelo financiamento desta pesquisa desenvolvida no âmbito do Projeto Rede de Monitoramento de Eventos Extremos da Amazônia - REMAM (Processo ${ }^{\circ}$ 01.09.0573.00).

\section{REFERÊNCIAS BIBLIOGRÁFICAS}

ALCANTARA, C. R.; SILVA DIAS, M.A.F.; SOUZA, E.P.; COHEN, J.C.P. Verification of the role of the low level jets in Amazon squall lines. Atmospheric Research, v.100, p. 36-44, 2011.

ASNANI, G. C. Tropical Meteorology, V. I-II, G. C. Asnani, Pune, India, pp. 603 and pp. 598, 1993.

BETTS, A.K. Convection in the tropics. Meteorology over the tropical oceans. Royal Meteorological. Society., Bracnell, England, p. 105-132, 1978.

CLIMANÁLISE. http://www.cptec.inpe.br/products/ climanalise. Abril, 2009.

CHONG, M.; AMAYENC, P.; SCIALOM, G.; TESTUD, J. A tropical squall line observed during the COPT 81 experiment in West Africa. Part I: Kinematic structure inferred from dual-Doppler radar data. Monthly Weather Review, v. 115, p. 670-694, 1987.

COHEN, J.C.P. Um Estudo observacional de linhas de instabilidade na Amazônia. Dissertação de Mestrado em Meteorologia. São José dos Campos, INPE, 1989.

COHEN, J.C.P.; CAVALCANTI, I.F.A.; BRAGA, R.H.M. Linhas de instabilidade na costa N-NE da América do Sul. In. CAVALCANTI, I.F.A.; FERREIRA, N.J.; SILVA, M.G.A.J.; DIAS, M.A.F.S. (Orgs.). Tempo e clima no Brasil. São Paulo: Oficina de Texto, 2009. P. 75-92.

COHEN, J.C.P., SILVA DIAS, M.A.F., NOBRE, C.A. Environmental conditions associated with Amazonian squall lines: a case study. Monthly Weather Review, v. 123, p. 3163-3174, 1995.

DIXON, M., WIENER G. TITAN: Thunderstorm, Identification, Tracking, Analysis and Nowcasting. Journal of Atmospheric and Oceanic Technology, 1993, v.10 (3), p.785-797.

DROSDOWSKY, W.; HOLLAND, G.J. North Australian cloud lines. Monthly Weather Review, v. 115, p. 2645-2659, 1987.

GAMACHE, J.F.; HOUZE, R.A.J. Mesoscale air motions associated with a squall line. Monthly Weather Review, v. 110, p. 118-135, 1982.

GANDU, A.W.; SILVA DIAS, P. L. Impact of tropical heat sources on the South American tropospheric upper circulation and subsidence. Journal of Geophysics Research, v. 103, p. 6001-60015, 1998.

GRAY, W. M.; JACOBSON JR, R. W. Diurnal variation of deep cumulus convection. Monthly Weather Review, v. 105, p. 104-188, 1977.

GRIMM, A. M., SILVA DIAS, P. L. Analysis of tropicalextratropical interactions with influence functions of a barotropic model. Journal of Atmospheric Sciences. v. 52, p. 3538-3555, 1995.

HOUZE JR, R.A. Structure and dynamics of a tropical squall line system. Monthly Weather Review, v. 105, p. 1540 $1567,1977$.

HOUZE JR, R.A.; RAPPAPORT, E.N. Air motions and precipitation structure of an early summer squall line over the Eastern tropical Atlantic. Journal of the Atmospheric Sciences, v. 41, p. 553-574, 1984.

INSTITUTO BRASILEIRO DE GEOGRAFIA E ESTATÍSTICA- IBGE Censo agropecuário - 2006: Brasil, Grandes Regiões e Unidades da Federação. Disponível em: $<$ http://www.ibge.gov.br >

KOUSKY, V.E. Pentad outgoing longware radiation climatology for the South American sector. Revista Brasileira de Meteorologia, v. 3, p. 217-231, 1988.

MARENGO, J. A.; B. LIEBMANN; V. E. KOUSKY; N. P. FILIZOLA; I. C. WAINER. Onset and end of the rainy 
season in the Brazilian Amazon basin. Journal of Climate, v. 14 , p. $833-852.2001$.

MOLION, L.C.B. Climatologia Dinâmica da Região Amazônica: Mecanismos de Precipitação. Revista Brasileira de Meteorologia, v. 2, p. 107-117, 1987.

MOLION, L. C. B. Amazonian rainfall and its variability. In: Hydrology and Water Management in the Humid Tropics, Ed., p.p. 99-111, Cambridge University Press, Cambridge, 1993.

NOBRE, C.A. Tropical heat sources and their associated largescale atmospheric circulation. Anais... First Conference on Southern hemisphere meteorology. Preprint Volume. São José dos Campos/SP. 1983. 104-107.

RAO, V.B.; HADA, K. Characteristics of rainfall over Brazil: annual variations and connections with southern oscillation. Theoretical and Applied Climatology, v. 42, p. 81-91, 1990.

RAO, M.V.; PALIYATH, G.; ORMROD, D.P. Ultraviolet-Band ozone-induced biochemical changes in antioxidant enzymes of Avabi-dopsis thaliana. Plant Physiol, v. 110, p. 125-136, 1996.
TOKAY, A.; SHORT, D. A. Evidence from tropical raindrop spectra of the origin of rain from stratiform and convective clouds, Journal of Applied Meteorology, v. 35, p. 355371, 1996.

TOMASELLA, J.; BORMA, L. S.; MARENGO, J. A.; RODRIGUEZ, D. A.; CUARTAS, L. A.; NOBRE, C.A.; PRADO, M.C.R. . The droughts of 1996-97 and 2004-05 in Amazonia: Hydrological response in the river main-stem. Hydrological Processes (Print), 2010.

XAVIER, T. M. B. S.; XAVIER, A. F. S.; DIAS, P. L. S.; DIAS, M. A. F. S. A Zona de Convergência Intertropical - ZCIT e suas relações com a chuva no Ceará. Revista Brasileira de Meteorologia, v. 15, n. 1, p. 27-43, 2000.

WALLACE, J. M. Diurnal variations in precipitation and thunderstorm frequency over the conterminous United States. Monthly Weather Review, v. 103, p. 406-419, 1975. 